\title{
Cancer Stem Cells and the Unicellular Life Cycle of Cancer
}

ISSN: 2637-773X

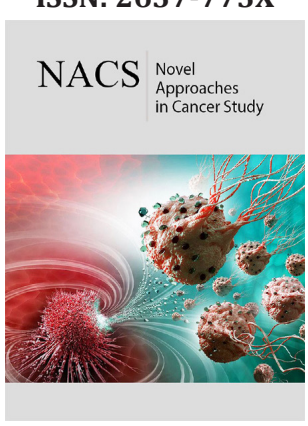

*Corresponding author: Vladimir F Niculescu, Cell biologist, Kirschenweg 1, 86420 Diedorf, Germany,

Email: vladimirniculescu@yahoo.com

Submission: 鲇 October 21, 2019

Published: 漹October 24, 2019

Volume 3 - Issue 3

How to cite this article: Niculescu VF (2019). Cancer Stem Cells and the Unicellular Life Cycle of Cancer. Nov Appro in Can Study. 3(3). NACS.000565.2019. DOI: 10.31031/NACS.2019.03.000565

Copyright@ Vladimir F Niculescu, This article is distributed under the terms of the Creative Commons Attribution 4.0 International License, which permits unrestricted use and redistribution provided that the original author and source are credited.

\author{
Vladimir F Niculescu*
}

Cell biologist, Germany

\begin{abstract}
All eukaryotes, from protists to mammalians, preserve a unicellular life cycle inherited from the common ancestor that can be reactivated under unfavorable living conditions. The cell-of-origin of cancer escapes its death by forming a protected polyploid cyst-like structure (CLS), that starts the unicellular life cycle of cancer. The reversal to unicellularity occurs through genomic and epigenetic alterations that activate the MUT switch of early Metazoans and not through mutations. The microcell progeny of CLSs spread into tissues and organs and form the CSC pool of aCLS cancers. Depending on the environment, the CSC pool differentiates a reproductive cell subline, which forms new aCLSs by cyclic encystment and asymmetric cell division, or a somatic subline, which proliferates strongly by symmetric cell division without cyst differentiation.
\end{abstract}

Keywords: Oncogenic transformation; Reversal to unicellularity; Tumorigenic pathogen; Microcells (germ cells); Resistance; Relapse

Abbreviations: MUT: Multicellular-Unicellular Transition; CLS: Cyst-like Structures; CSC: Cancer Stem Cell; PGCC: Polyploid Giant Cancer Cell; SCP: Somatic Stem Cell Precursor

\section{Opinion}

Does cancer stem cells (CSCs) really originate from abnormal human stem cells (hSCs)? And is cancer a disease of cell differentiation in multicellular organisms or does it have deeper roots in the evolutionary history of life? Cancer stem cells were found in tumors of various tissues and organs as well as in established tumor cell lines. Like normal stem cells, CSCs are capable of self-renewal, quiescence, and cell differentiation. They invade other tissues and cause secondary tumors, metastases and cancer recurrence. CSCs express the embryonic stem cell markers OCT4, NANOG, SOX2 and YAP [1]. All these findings led previous cancer researchers to the assumption that CSCs arise from deficient human stem cells (hSCs). Some cancer researchers regarded cancer as a "stem cell disease" [2].

In recent years, it has become apparent that tumor CSCs are closely related to another selfrenewing participant of the tumor mass, namely the reproductive cyst-like structure aCLS [3] often referred to as PGCC [4]. The polyploid aCLS (PGCC) is a cyst-like structure protected by an actin fiber sheath. It forms numerous monoploid 1C daughter cells (microcells, "germ" cells) by reductive nuclear division and asymmetric cellularization (non- mitotic cell division) and daughter cell dissemination. Microcells spread in various tissues and form metastases. Tumor hypoxia favors the formation of reproductive aCLSs. aCLSs and their offspring have CSC properties; they express cancer cell markers such as CD133 and CD44. The progeny of aCLSs have multipotent stemness and are capable to differentiate multiple cell lineages [1]. . Amend [5] considers the aCLS as a key actuator for tumorigenesis, metastasis and therapy resistance. A few months ago, Liu J [1] introduced the term "giant cell life cycle" to describe the developmental pathway between two consecutive aCLSs.

We recently introduced the term aCLS-cancers for a subset of cancers ( $\geq 37 \%$ of all acquired solid cancers) that forms aCLSs [6] and believe that the CSCs of aCLS cancers originate from the disseminating microcell progeny. In our opinion, the microcell progeny ("germ cells") are the precursors of CSCs. Also, we regard the "giant cell life cycle" as a unicellular (reproductive) cancer life cycle that begins with the oncogenic transformation of the cell-of- origin of cancer.

Based on our experience with unicellular/protist life cycles [7] we have been taking this idea for several years. We believe that all eukaryotes (including mammals and humans) conserve an ancestral unicellular life cycle that can be reactivated under stress and non- 
optimal living conditions. It is a process of reversal to unicellularity developed by unstable early Metazoans of the transition period. Non-optimal living conditions for multicellular life would reverse cells of the multicellular community to a unicellular lifestyle (MUT). Since reversal processes occur frequently at this time, early Metazoans have developed a molecular transition switch (MUT switch) that was taken over in the genome of all Metazoans and opens the gates to cancer [6].

Accordingly, the metabolically stressed cell-of-origin reactivates the MUT pathway switching into a primary, pretumorigenic pCLS. The oncogenic transformation occurs by genomic and epigenetic changes that are not driver mutations in the sense of certain DNA defects or DNA damage. The pCLS that initiates the unicellular life cycle of cancer is a home-grown tumorigenic pathogen of unicellular organization and lifestyle. It generates pretumorigenic pCSCs that disseminate in tissues and organs differentiating both reproductive and somatic cell clones (sublines). Intestinal amebae, that live in oxygen gradients between 0.1 and $<6 \%$ oxygen content, have a similar stem cell lineage. The microcell progeny of the amoeba cysts form the stem cell pool of amoebae, that differentiate environmental dependant either a reproductive or a vegetative subline; the reproductive non-pathogenic subline ("germ" cell subline) forms continuously cysts by asymmetric cell division and cyclic differentiation (ACD cysts) while the vegetative subline proliferates in intestine and organs by symmetric cell division without cyst formation. Under unfavorable living conditions, vegetative amoebae stop their mitotic cell cycle and form ICD cysts (induced cyst differentiation). The same happens during aCLS cancer development. Some of the somatic mutants (somatic stem cell precursors, SCPs) express the hidden differentiation potential of all cancer cells (aCLS differentiation potential) and generate new reproductive clones. In this way, SCPs transfer genomic and epigenetic modifications from the somatic to the reproductive cell subline that forms tumorigenic or metastatic aCLSs and numerous malignant tCSCs and mCSCs.

aCLS cancers are reproductive cancers and all cancer cells including CSCs are descendants of the pCLS lineage. The disseminating microcell offspring ("germ cells") proliferate into CSC pools that differentiate environmental dependant all somatic and reproductive sublines and clones. For the cells of the reproductive subline, we have proposed the term "stem cell family" [7]. It is subjected to the control mechanisms of unicellular cell line surveillance. Under hypoxic conditions, the reproductive subline forms numerous aCLS by fast cycling and asymmetric mitotic proliferation. Under conditions with increased oxygen content, the reproductive subline cycles slower. Genomically, the reproductive subline is protected by a functional G1 checkpoint, while somatic cells - with a defective G1 checkpoint - undergo replication errors and mutations. Microcells ("germ cells") and their DNA are protected by the actin fiber envelope of the aCLS and by a perinuclear layer of hypoxic rest mitochondria. They survive chemotherapy and give rise to new CSCs capable of metastases and recurrence (tCSCs, mCSCs). Cysts and cyst-like structures protect the offspring and increase their resistance to adverse living conditions and therapeutics. The unicellular life cycle of aCLS cancer to form uses the ancestral process of encystation and excystation of protists to form resistant cancer "germ cells" (microcells) and CSCs. Reproductive cyst formation is a well-known process of asexual reproduction and unicellular stem cell formation.

\section{References}

1. Liu J (2019) The "life code": A theory that unifies the human life cycle and the origin of human tumors. Seminars in Cancer Biology, pii: S1044579X (19): 30053-30057.

2. Basak U, Dutta A, Dutta Chowdhury D, Bhattacharya A, Banerjee S (2017) Deciphering the cancer puzzle: Cancer stem cells being the pivotal piece. J Stem Cell Res Transplant 4(1): 1025.

3. Niculescu VF (2018) Carcinogenesis: Recent insights in protist stem cell biology lead to a better understanding of atavistic mechanisms implied in cancer development. MOJ Tumor Res 1(1): 00004.

4. Zhang S, Mercado-Uribe I, Xing Z, Sun B, Kuang J, et al. (2014) Generation of cancer stem-like cells through the formation of polyploid giant cancer cells. Oncogene 33(1): 116-128.

5. Amend SR, Torga G, Lin KC, Kostecka LG, de Marzo A, et al. (2019) Polyploid giant cancer cells: Unrecognized actuators of tumorigenesis, metastasis, and resistance. Prostate 79(13): 1489-1497.

6. Niculescu VF (2019) aCLS cancers: genomic and epigenetic changes transform the cell of origin of cancer into a tumorigenic pathogen of unicellular organization and lifestyle. Gene 144174.

7. Niculescu VF (2018) The cancer stem cell family: Atavistic origin and tumorigenic development. MOJ Tumor Res 1(2): 71-74. 